\title{
Cryptanalysis of a Fuzzy Identity Based Encryption Scheme in the Standard Model
}

\author{
$\mathrm{Xu}$ An WANG ${ }^{1}$, Xiaoyuan $\mathrm{YANG}^{1}$, Minqing $\mathrm{ZHANG}^{1}$, Yong $\mathrm{YU}^{2}$ \\ ${ }^{1}$ Key Laboratory of Information and Network Security \\ Engineering University of Chinese Armed Police Force \\ $X i$ 'an 710086, P.R. China \\ ${ }^{2}$ School of Computer Science and Engineering \\ University of Electronic Science and Technology of China \\ Chengdu 610054, P.R. China \\ e-mail:wangxahq@yahoo.com.cn
}

Received: October 2010; accepted: December 2011

\begin{abstract}
Fuzzy identity based encryption (FIBE), proposed by Sahai and Waters, is a new kind of identity based encryption. It allows users with identity $w$ can decrypt ciphertext for $w^{\prime}$ if and only if $w$ is close enough to $w^{\prime}$. Recently, Ren et al. proposed a new FIBE scheme and claimed it is fully CCA2 secure in the standard model with a tight reduction. However, in this paper we will show that their scheme is not correct. Furthermore, the key generation process of their scheme cannot resist the collusion attack, which is a basic security requirement for FIBE. At last, we propose a new fully secure FIBE scheme based on the Sahai-Waters FIBE scheme and prove its security by using the "dual system encryption" technique.
\end{abstract}

Keywords: cryptography, fuzzy identity based encryption, fully secure, CCA2-secure, attack.

\section{Introduction}

Shamir (1984) introduced the concept of identity based encryption (IBE), whose motivation is to ease the certificate management. A user's public key in an identity based system is some unique information about the identity of the user (e.g., email address). However, only in 2001 the first practical identity based encryption was realized by Boneh and Franklin using bilinear maps on elliptic curve. However, their scheme can only be proved secure in the random oracle model. In Eurocrypt'03, Canetti et al. proposed a weaker security notion, selective identity (selective-ID) security for IBE, relative to which they were able to build an inefficient but secure IBE scheme in the standard model (Canetti et al., 2003). In Eurocrypt'04, Boneh and Boyen proposed two new efficient selective identity secure identity based encryption schemes without random oracles $\left(B B_{1} I B E\right.$ and $\mathrm{BB}_{2}$ IBE) (Boneh and Boyen, 2004a). In Crypto'04, they improved their scheme to full security (Boneh and Boyen, 2004b) but with a loose security reduction. In Eurocrypt'05, Waters improved their work by proposing a fully secure identity based encryption with tight security proof in the standard model (Waters' IBE; Waters, 2005). In Eurocrypt'06, 
Gentry gave another interesting efficient fully secure identity based encryption with tight security proof in the standard model but based on a strong assumption (Gentry's IBE; Gentry, 2006).

\subsection{Fuzzy Identity Based Encryption}

In Eurocrypt'05, Sahai and Waters introduced a new concept, fuzzy identity based encryption (FIBE), which aimed at error-tolerance property of IBE (Sahai and Waters, 2005). In a FIBE, a user with the secret key for the identity $w$ is able to decrypt a ciphertext encrypted with the public key $w^{\prime}$ if and only if $I D$ and $I D^{\prime}$ are within a certain distance of each other as judged by some metric. FIBE at least has two interesting applications, the first is an IBE system with biometric identities, the error-tolerance property of FIBE allows for a private key (derived from a measurement of a biometric ) to decrypt a ciphertext encrypted with a slightly different measurement of the same biometric. Secondly FIBE can be used for "attribute-based encryption (ABE)", where both ciphertexts and secret keys are associated with sets of attributes. Decryption is enabled if and only if the cipertext and secret key attribute sets overlap by at least a fixed threshold value d. Goyal et al. (2006) proposed an ABE scheme that provides fine-grained sharing of encrypted data. Piretti et al. (2006) used FIBE to realize their secure information management architecture. Later Baek et al. constructed two new efficient FIBE schemes in the random oracle model (Baek et al., 2007).

\subsection{Dual System Encryption}

In Crypto'09, Waters (2009) introduced a new methodology named "dual system encryption" for proving security of encryption systems. In this system, both ciphertexts and private keys can take on one of two indistinguishable forms, semi-functional one or normal one. A semi-functional private key will be able to decrypt all normally generated ciphertexts; however, a semi-functional private key cannot decrypt a semi-functional ciphertext. The security can be reduced to a sequence of games, where the challenge ciphertext and the private keys one by one were changed to be semi-functional. In the finally game, the challenge ciphertext and all private keys are semi-functional, at which point the adversary can only guess the challenge plaintext randomly. In TCC'10, Lewko and Waters 2010b) realized the dual system encryption in the composite order group and thus achieve fully secure IBE and HIBE scheme with simple structure. In Eucrypto'10, Lewko et al. (2010) construct the fully secure $A B E$ also by using dual system encryption technique.

\subsection{Our Contribution}

Recently Ren et al. (2010) claimed to construct a fully CCA2 secure FIBE in the standard model with a tight reduction. However, we will show that their scheme is not correct at all. Furthermore, the key generation process of their scheme cannot resist the collusion attack, which is crucial for FIBE. As an improvement of Ren et al. 's FIBE result, we propose a new fully secure FIBE scheme based on the Sahai-Waters FIBE scheme and prove its security by using the "dual system encryption" technique. 


\subsection{Organization}

The organization of this paper is as follows. Section 2 gives the definition and security model for FIBE. We review Ren et al.'s scheme and show their scheme is not correct and secure at all in Section 3. In Section 4, we propose our new FIBE scheme and prove its security. We conclude our paper in the last section.

\section{Definition and Security Model for FIBE}

\subsection{Definition}

A FIBE consists of the following algorithms.

1. Setup $\left(1^{k}\right)$. Taking $1^{k}$ as the security parameter, the Private Key Generator (PKG) runs this algorithm to generate its master key $m k$ and public parameters params which contains an error tolerance parameter $d$. Note that params is given to all interested parties while $m k$ is kept secret.

2. Extract $(m k$, ID). Taking the master key $m k$ and an identity ID as input, the PKG runs this algorithm to generate a private key associated with ID, denoted by $d_{\mathrm{ID}}$.

3. Encrypt (params, ID', M). Taking the public parameters params, an identity ID', and a plaintext $M$ as input, a sender runs this algorithm to generate a ciphertext $C^{\prime}$.

4. Decrypt $\left(\right.$ params, $\left.d_{\mathrm{ID}}, C^{\prime}\right)$. Taking the public parameters params, a private key $d_{\mathrm{ID}}$ associated with the identity ID and a ciphertext $C$ encrypted with an identity ID such that $\left|\mathrm{ID}^{\prime} \cap \mathrm{ID}\right|>d$ as input, a receiver runs this algorithm to get a decryption, which is either a plaintext or a "Reject" message.

\subsection{Security Model}

(IND-FID-CCA2 and IND-FID-CPA Security.) The semantic security against an adaptive chosen ciphertext attack security for a fuzzy IBE system is defined by the following game between an adversary and a challenger.

Setup. The challenger runs algorithm Setup, and forwards parameters to the adversary. Phase 1. Proceeding adaptively, the adversary issues queries $q_{1}, \ldots, q_{m}$, where $q_{i}$ is one of the following:

- Key generation query $\left\langle\mathrm{ID}_{i}\right\rangle$ The challenger runs algorithm KeyGen on $\mathrm{ID}_{i}$ and forwards the resulting private key to the adversary.

- Decryption query $\left\langle\mathrm{ID}_{i}, c_{i}\right\rangle$ The challenger runs algorithm KeyGen on $\mathrm{ID}_{i}$, decrypts $c_{i}$ with the resulting private key, and sends the result to the adversary.

Challenge. The adversary sends $\left(I^{*}, m_{0}, m_{1}\right)$ to the challenger, where $\mid$ ID $\cap I^{*} \mid<d$, and ID denotes the identity that has appeared in key generation and decryption query in Phase 1 . The challenger selects a random bit $k \in\{0,1\}$, sets $c^{*}=\operatorname{Encrypt}\left(\right.$ params $\left., \mathrm{ID}^{*}, m_{k}\right)$, and sends $c^{*}$ to the adversary as its challenged ciphertext. 
Phase 2. $\mathcal{A}$ executes the following queries:

- Key generation query $\langle\mathrm{ID}\rangle$, where $\left|\mathrm{ID} \cap \mathrm{ID}^{*}\right|<d$.

- Decryption query $\langle\mathrm{ID}, c\rangle$, where $c \neq c^{*}$.

These queries maybe be adaptive.

Guess. The adversary submits a guess $k^{\prime} \in\{0,1\}$.

We call an adversary $\mathcal{A}$ in the above game an IND-FID-CCA2 adversary. The advantage of $\mathcal{A}$ is defined as $\left|\operatorname{Pr}\left[k=k^{\prime}\right]-\frac{1}{2}\right|$.

Definition 1. A fuzzy IBE system is $\left(t, \epsilon, q_{k}, q_{d}\right)$ IND-FID-CCA2 secure if all $t$-time IND-FID-CCA2 adversaries making at most $q_{k}$ key generation queries and $q_{d}$ decryption queries have advantage of at most $\epsilon$ in the above game.

We call an adversary $\mathcal{A}$ an IND-FID-CPA adversary if the adversary remain the same in the above game except it cannot execute decryption queries. The advantage of $\mathcal{A}$ is defined as $\left|\operatorname{Pr}\left[k=k^{\prime}\right]-\frac{1}{2}\right|$.

DEFINITION 2. A fuzzy IBE system is $\left(t, \epsilon, q_{k}\right)$ IND-FID-CPA secure if all $t$-time INDFID-CCA2 adversaries making at most $q_{k}$ key generation queries have advantage of at most $\epsilon$ in the above game.

Collusion Attack Security. Collusion attack for a fuzzy IBE system is defined by the following game between an adversary and a challenger.

Setup. The challenger runs algorithm Setup, and forwards parameters to the adversary.

Phase 1. Proceeding adaptively, the adversary issues queries $q_{1}, \ldots, q_{m}$, where $q_{i}$ is one of the following:

- Key generation query $\left\langle\mathrm{ID}_{i}\right\rangle$ The challenger runs algorithm KeyGen on $\mathrm{ID}_{i}$ and forwards the resulting private key to the adversary.

Challenge. The adversary can output a valid private key of ID* which is not equal any of $\operatorname{ID}_{i}(i=1 \ldots, m)$

We call the adversary successfully run the collusion attack on the scheme.

\section{Cryptanalysis of Ren et al.'s FIBE Scheme}

\subsection{Review of Ren et al.'s FIBE Scheme}

Assume an identity ID $=\left(I D_{1}, I D_{2}, \ldots, I D_{n}\right)$, where $n$ is the length of ID and $I D_{i} \in Z_{p}^{*}$ represents the minimal error tolerance and $n>d$. Now we wish to create a FIBE scheme in which a ciphertext created using identity ID' can be decrypted only by a private key associated with identity ID, where $\left|\operatorname{ID} \cap \mathrm{ID}^{\prime}\right|>d$. We also define the Lagrange coefficient $\Delta_{i, S}$ for $i \in Z_{p}^{*}$ and a set $S$, of elements in $Z_{p}^{*}: \Delta_{i, S}(x)=\prod_{j \in S, j \neq i} \frac{x-j}{i-j}$. 
Setup. Let $p$ be a large prime number, $G_{1}, G_{2}$ are groups of order $p$. $e: G_{1} \times G_{1} \rightarrow G_{2}$ is a bilinear map, $g$ is a generator of $G_{1}, h:\left(Z_{p}^{*}\right)^{\{0,1\}} \times\{1,2, \ldots, n\} \rightarrow$ $Z_{p}^{*}, H: G_{1}^{n} \times G_{2}^{l} \rightarrow Z_{p}^{*}$ are collision-resistant hash functions, where $l \in$ $Z_{p}^{*}$. The PKG randomly choose $\alpha \in Z_{p}^{*}, h_{0}, h_{1}, h_{2} \in G_{1}$, and two random polynomials $f(x), q(x) \in Z_{p}^{*}[x]$ of degree 1 and $d-1$ respectively, where $f(x)=a x+b$. If $h_{0}=h_{2}^{-a}$ or $h_{1}=h_{2}^{-b}$, randomly choose $f(x)$ again. The $P K G$ computes $g_{1}=g^{\alpha}, g_{2}=g^{q(0)}, g_{3}=g_{1}^{q(0)}$. The public parameters are $\left(g, g_{1}, g_{2}, g_{3}, h_{0}, h_{1}, h_{2}, d, h, H, f(x)\right)$ and $\alpha, q(x)$ are the private keys of PKG.

KeyGen. To a user $U$ with identity ID $=\left(I D_{1}, I D_{2}, \ldots, I D_{n}\right)$, the PKG randomly chooses $r_{0} \in Z_{p}^{*}$ and computes

$$
d_{0}=r_{0}, d_{i}=\left(h_{0} h_{1}^{r_{0}} h_{2}^{f\left(r_{0}\right)}\right)^{\frac{\alpha q(i)}{q(0) h\left(I D_{i}, i\right)+h(i)}} \quad(i=1,2, \ldots, n)
$$

so the private key of $U$ is $d_{\mathrm{ID}}=\left(d_{0}, d_{1}, d_{2}, \ldots, d_{n}\right)$.

Encrypt. To encrypt a message $m \in G_{2}$ with a key associated with identity ID $^{\prime}=$ $\left(I D_{1}^{\prime}, \ldots, I D_{n}^{\prime}\right)$, randomly choose $s \in Z_{p}^{*}$ and a polynomial $A(x) \in Z_{p}^{*}[x]$ of degree $d-1$, compute:

$$
\begin{aligned}
& u_{i}=\left(g_{2}^{h\left(I D_{i}^{\prime}, i\right)} \cdot g^{h(i)}\right)^{s A(i)}(i=1,2, \ldots, n), \quad v_{1}=e\left(g_{3}, h_{1}\right)^{s A(0)}, \\
& v_{2}=e\left(g_{3}, h_{2}\right)^{s A(0)}, \quad w=m \cdot e\left(g_{3}, h_{0}\right)^{s A(0)+\gamma}, \\
& \beta=H\left(u_{1}, \ldots, u_{n}, v_{1}, v_{2}, w, m \cdot e\left(g_{3}, h_{0}\right)^{s A(0)}\right),
\end{aligned}
$$

where $\gamma=H\left(u_{1}, \ldots, u_{n}, v_{1}, v_{2}, e\left(g_{3}, h_{0}\right)^{s A(0)}\right)$. The ciphertext of message $m$ is $c=\left(u_{1}, \ldots, u_{n}, v_{1}, v_{2}, w, \beta\right)$.

Decrypt. Suppose that a ciphertext $c$ is encrypted with a key associated with identity ID' and we have a private key for identity ID, where $\left|I D \cap I D^{\prime}\right|>d$. Choose an arbitrary $d$-element subset $S=\left\{i \mid i \in\{1, \ldots, n\}, I D_{i} \in \mathbf{I D} \cap \mathbf{I D}^{\prime}\right\}$ and decrypt

$$
\begin{aligned}
& \frac{\prod_{i \in S} e\left(u_{i}, d_{i}\right)^{\Delta_{i, S}(0)}}{v_{1}^{d_{0}} v_{2}^{f\left(d_{0}\right)}}=e\left(g_{3}, h_{0}\right)^{s A(0)}, \\
& \gamma=H\left(u_{1}, \ldots, u_{n}, v_{1}, v_{2}, e\left(g_{3}, h_{0}\right)^{s A(0)}\right), \\
& \frac{w}{e\left(g_{3}, h_{0}\right)^{\gamma}}=R, \beta^{\prime}=H\left(u_{1}, \ldots, u_{n}, v_{1}, v_{2}, w, R\right),
\end{aligned}
$$

and verify whether $\beta^{\prime}=\beta$. If yes, decrypt $\frac{R}{e\left(g_{3}, h_{0}\right)^{s A(0)}}=m$. Otherwise, return an error message. 


\subsection{On the Correctness}

In Ren et al. (2010), the correctness of the new fuzzy IBE scheme is shown as follows where $I D_{i} \in \mathrm{ID} \cap \mathrm{ID}^{\prime}$ if $i \in S$.

$$
\begin{aligned}
& e\left(u_{i}, d_{i}\right)=e\left(\left(g_{2}^{h\left(I D_{i}^{\prime}, i\right)} \cdot g^{h(i)}\right)^{s A(i)},\left(h_{0} h_{1}^{r_{0}} h_{2}^{f\left(r_{0}\right)}\right)^{\frac{\alpha \cdot q(i)}{q(0) h\left(I D_{i}, i\right)+h(i)}}\right) \\
& =e\left(g^{s A(i)\left(q(0) h\left(I D_{i}, i\right)+h(i)\right)},\left(h_{0} h_{1}^{r_{0}} h_{2}^{f\left(r_{0}\right)}\right)^{\frac{\alpha \cdot q(i)}{q(0) h\left(I D_{i}, i\right)+h(i)}}\right) \\
& =e\left(g^{s A(i)},\left(h_{0} h_{1}^{r_{0}} h_{2}^{f\left(r_{0}\right)}\right)^{\alpha q(i)}\right), \\
& \prod_{i \in S} e\left(u_{i}, d_{i}\right)^{\delta_{i, S}(0)} \\
& =\prod_{i \in S} e\left(\left(g^{s A(i)}, h_{0} h_{1}^{r_{0}} h_{2}^{f\left(r_{0}\right)}\right)^{\alpha q(i)}\right)^{\delta_{i, S}(0)} \\
& =e\left(g_{1}^{s}, h_{0} h_{1}^{r_{0}} h_{2}^{f\left(r_{0}\right)}\right)^{\Sigma_{i \in S} A(i) q(i) \delta_{i, S}(0)} \\
& =e\left(g_{1}^{s}, h_{0} h_{1}^{r_{0}} h_{2}^{f\left(r_{0}\right)}\right)^{A(0) q(0)} \\
& =e\left(g_{3}, h_{0}\right)^{s A(0)} e\left(g_{3}, h_{1}\right)^{s r_{0} A(0)} e\left(g_{3}, h_{2}\right)^{s f\left(r_{0}\right) A(0)}, \\
& \frac{\prod_{i \in S} e\left(u_{i}, d_{i}\right)^{\delta_{i, S}(0)}}{v_{1}^{d_{0}} v_{2}^{f\left(d_{0}\right)}}=e\left(g_{3}, h_{0}\right)^{s A(0)}, \\
& \gamma=H\left(u_{1}, \ldots, u_{n}, v_{1}, v_{2}, e\left(g_{3}, h_{0}\right)^{s A(0)}\right), \\
& \frac{w}{e\left(g_{3}, h_{0}\right)^{\gamma}}=m \cdot e\left(g_{3}, h_{0}\right)^{s A(0)}=R, \\
& \beta^{\prime}=H\left(u_{1}, \ldots, u_{n}, v_{1}, v_{2}, w, R\right)=\beta, \quad R / e\left(g_{3}, h_{0}\right)^{s A(0)}=m .
\end{aligned}
$$

But actually, the equation of

$$
e\left(g_{1}^{s}, h_{0} h_{1}^{r_{0}} h_{2}^{f\left(r_{0}\right)}\right)^{\Sigma_{i \in S} A(i) q(i) \delta_{i, S}(0)}=e\left(g_{1}^{s}, h_{0} h_{1}^{r_{0}} h_{2}^{f\left(r_{0}\right)}\right)^{A(0) q(0)}
$$

cannot hold.

In the reconstruction of an interpolating polynomial, $n$ point-values are required to reconstruct a polynomial with degree $n-1$ to satisfy these $\mathrm{n}$ point-values. The degree of $A(x) q(x)$ is the sum of the degrees of $A(x)$ and $q(x)$, which is $2 d-2$. But $S=\{i \mid i \in$ $\left.\{1, \ldots, n\}, I D_{i} \in \mathbb{I D} \cap I^{\prime}\right\}$ is a $d$-element subset, which is not $2 d-1$, meaning the above equation cannot hold at all. Thus the Decrypt algorithm is not correct.

\subsection{Collusion Attack}

Ren et al. claimed that their FIBE scheme is IND-FID-CCA secure, However, in this section, we show that this is not true. Concretely, there exists a polynomial time adversary $\mathcal{A}$ who can act the collusion attack against the FIBE scheme. Adversary $\mathcal{A}$ works as follows: 
1. In Setup phase, adversary $\mathcal{A}$ obtains the public parameters params from the challenger.

2. In Phase 1 and Challenge phase, adversary $\mathcal{A}$ chooses a target identity ID* $=$ $\left(I D_{1}^{*}, \ldots, I D_{n}^{*}\right)$, denote

$$
\begin{aligned}
& \widehat{\mathbf{I D}}_{1}=\left(I D_{1}^{*}, I D_{21}, \ldots, I D_{n 1}\right), \\
& \widehat{\mathbf{I D}}_{2}=\left(I D_{12}, I D_{2}^{*}, \ldots, I D_{n 2}\right), \\
& \ldots \ldots \ldots \ldots \ldots \ldots \\
& \widehat{I D}_{n}=\left(I D_{1 n}, I D_{2 n}, \ldots, I D_{n}^{*}\right),
\end{aligned}
$$

where $I D_{1 i} \neq I D_{1}^{*}(i=2,3, \ldots, n), I D_{2 i} \neq I D_{2}^{*}(i=1,3, \ldots, n), \ldots, I D_{n i} \neq$ $I D_{n}^{*}(i=1,2, \ldots, n-1)$. Note here $\left|\widehat{I D}_{1}, \widehat{I D}_{2}, \ldots,\right| \mathbf{I}_{n}$ all satisfies $|\mathbf{I D} \cap| \mathbf{I D}^{*} \mid<d$.

(a) First adversary $\mathcal{A}$ issues key generation queries on every $\widehat{I D}_{i}(i=1, \ldots, n)$ two times, he will get the private keys as follows:

$$
\begin{aligned}
& d_{\widehat{\mathbb{D}}_{1}}^{0}=\left\{d_{01}^{0}=r_{1}^{0}, d_{11}^{0}=\left(h_{0} h_{1}^{r_{1}^{0}} h_{2}^{f\left(r_{1}^{0}\right)}\right)^{\frac{\alpha(0) h\left(I D_{1}^{*}, 1\right)+h(1)}{q(1)}},\right. \\
& \left.d_{i 1}^{0}=\left(h_{0} h_{1}^{r_{1}^{0}} h_{2}^{f\left(r_{1}^{0}\right)}\right)^{\frac{\alpha q(i)}{q(0) h\left(I D_{i 1}, i\right)+h(i)}}(i=2,3, \ldots, n)\right\}, \\
& d_{\widehat{\mathbb{D}}_{1}}^{1}=\left\{\left(d_{01}^{1}=r_{1}^{1}, d_{11}^{1}=\left(h_{0} h_{1}^{r_{1}^{1}} h_{2}^{f\left(r_{1}^{1}\right)}\right)^{\frac{\alpha q(1)}{q(0) h\left(I D_{1}^{*}, 1\right)+h(1)}},\right.\right. \\
& \left.d_{i 1}^{1}=\left(h_{0} h_{1}^{r_{1}^{1}} h_{2}^{f\left(r_{1}^{1}\right)}\right)^{\frac{\alpha q(i)}{q(0) h(I D i 1, i)+h(i)}}(i=2,3, \ldots, n)\right\}, \\
& d_{\widehat{\mathrm{ID}}_{2}}^{0}=\left\{d_{02}^{0}=r_{2}^{0}, d_{22}^{0}=\left(h_{0} h_{1}^{r_{2}^{0}} h_{2}^{f\left(r_{2}^{0}\right)}\right)^{\frac{\alpha(0) h\left(I D_{2}^{*}, 2\right)+h(2)}{q(2)}},\right. \\
& \left.d_{i 2}^{0}=\left(h_{0} h_{1}^{r_{2}^{0}} h_{2}^{f\left(r_{2}^{0}\right)}\right)^{\frac{\alpha q(i)}{q(0) h\left(I D_{i 2}, i\right)+h(i)}}(i=1,3, \ldots, n)\right\}, \\
& d_{\widehat{\mathbb{D}}_{2}}^{1}=\left\{d_{02}^{1}=r_{2}^{1}, d_{22}^{1}=\left(h_{0} h_{1}^{r_{2}^{1}} h_{2}^{f\left(r_{2}^{1}\right)}\right)^{\frac{\alpha q(2)}{q(0) h\left(I D_{2}^{*}, 2\right)+h(2)}},\right. \\
& \left.d_{i 2}^{1}=\left(h_{0} h_{1}^{r_{2}^{1}} h_{2}^{f\left(r_{2}^{1}\right)}\right)^{\frac{\alpha q(i)}{q(0) h\left(I D_{i 2}, i\right)+h(i)}}(i=1,3, \ldots, n)\right\}, \\
& d_{\widehat{\mathbb{D}}_{n}}^{0}=\left\{d_{0 n}^{0}=r_{n}^{0}, d_{n n}^{0}=\left(h_{0} h_{1}^{r_{n}^{0}} h_{2}^{f\left(r_{n}^{0}\right)}\right)^{\overline{q(0) h\left(I D_{n}^{*}, n\right)+h(n)}},\right. \\
& \left.d_{i n}^{0}=\left(h_{0} h_{1}^{r_{n}^{0}} h_{2}^{f\left(r_{n}^{0}\right)}\right)^{\frac{\alpha q(i)}{q(0) h\left(I D_{i n}, i\right)+h(i)}}(i=1,2, \ldots, n-1)\right\}, \\
& d_{\widehat{\mathbb{D}}_{n}}^{1}=\left\{d_{0 n}^{1}=r_{n}^{1}, d_{n n}^{1}=\left(h_{0} h_{1}^{r_{n}^{1}} h_{2}^{f\left(r_{n}^{1}\right)}\right)^{\frac{\alpha q(n)}{q(0) h\left(I D_{n}^{*}, n\right)+h(n)}},\right. \\
& \left.d_{i n}^{1}=\left(h_{0} h_{1}^{r_{n}^{1}} h_{2}^{f\left(r_{n}^{1}\right)}\right)^{\frac{\alpha q(n)}{q(0) h\left(I D_{i n}, i\right)+h(n)}}(i=1,2, \ldots, n-1)\right\},
\end{aligned}
$$


where in $d_{i j}^{t}, i$ denotes the place in the private key, $j$ denotes the place in the identity list $\left(\widehat{I D}_{1}, \widehat{I D}_{2}, \ldots, \widehat{I D}_{n}\right)$, and $t$ denotes the time the key generation query issued. $r_{1}^{0}, r_{1}^{1}, \ldots, r_{n}^{0}, r_{n}^{1}$ are all randomly chosen from $Z_{p}^{*}$.

(b) Note $f(x)=a x+b$ is a public key, thus adversary $\mathcal{A}$ can compute

$$
\begin{aligned}
d_{11}^{0} & =\left(h_{0} h_{1}^{r_{1}^{0}} h_{2}^{f\left(r_{1}^{0}\right)}\right)^{\frac{\alpha q(1)}{q(0) h\left(I D_{1}^{*}, 1\right)+h(1)}}, \\
& =\left(\left(h_{0} h_{2}^{b}\right)\left(h_{1} h_{2}\right)^{a r_{1}^{0}}\right)^{\frac{\alpha q(1)}{q(0) h\left(I D_{1}^{*}, 1\right)+h(1)}}, \\
d_{11}^{1} & =\left(h_{0} h_{1}^{r_{1}^{1}} h_{2}^{f\left(r_{1}^{1}\right)}\right)^{\frac{\alpha q(1)}{q(0) h\left(I D_{1}^{*}, 1\right)+h(1)}}, \\
& =\left(\left(h_{0} h_{2}^{b}\right)\left(h_{1} h_{2}\right)^{a r_{1}^{1}}\right)^{\frac{\alpha q(1)}{q(0) h\left(I D_{1}^{*}, 1\right)+h(1)}} .
\end{aligned}
$$

Adversary $\mathcal{A}$ can then have

$$
\begin{aligned}
& A_{1}=\left(\left(h_{1} h_{2}\right)^{a}\right)^{\frac{\alpha q(1)}{q(0) h\left(I D_{1}^{*}, 1\right)+h(1)}}=\left(\frac{d_{11}^{0}}{d_{11}^{1}}\right)^{\frac{1}{r_{1}^{0}-r_{1}^{1}}} \\
& B_{1}=\left(h_{0} h_{2}^{b}\right)^{\frac{\alpha q(1)}{q(0) h\left(I D_{1}^{*}, 1\right)+h(1)}}=\frac{d_{11}^{0}}{A_{1}^{r_{1}^{0}}}
\end{aligned}
$$

Similarly, adversary $\mathcal{A}$ can have

$$
\begin{array}{ll}
A_{2}=\left(\frac{d_{22}^{0}}{d_{22}^{1}}\right)^{\frac{1}{r_{2}^{0}-r_{2}^{1}}}, & B_{2}=\frac{d_{22}^{0}}{A_{2}^{r_{2}^{0}}} \\
\ldots \ldots \ldots & B_{n}=\frac{d_{n n}^{0}}{A_{n}^{r_{n}^{0}}}
\end{array}
$$

(c) Adversary $\mathcal{A}$ then randomly chooses $r \in Z_{p}^{*}$ and computes a valid private key for ID*

$$
\begin{aligned}
& \widetilde{d_{0}}=r, \widetilde{d_{1}}=B_{1}\left(A_{1}\right)^{r}=\left(h_{0} h_{1}^{r} h_{2}^{f(r)}\right)^{\frac{\alpha q(1)}{q(0) h\left(I D_{1}^{*}, 1\right)+h(1)}}, \\
& \widetilde{d_{2}}=B_{2}\left(A_{2}\right)^{r}=\left(h_{0} h_{1}^{r} h_{2}^{f(r)}\right)^{\frac{\alpha q(2)}{q(0) h\left(I D_{2}^{*}, 2\right)+h(2)}}, \ldots \ldots \ldots, \\
& \widetilde{d_{n}}=B_{n}\left(A_{n}\right)^{r}=\left(h_{0} h_{1}^{r} h_{2}^{f(r)}\right)^{\frac{\alpha q(n)}{q(0) h\left(I D_{n}^{*}, n\right)+h(n)}} .
\end{aligned}
$$

We can verify it is a valid private key for ID*.

Thus adversary $\mathcal{A}$ can successfully collusion attack this scheme. 


\section{New Fully Secure FIBE Scheme}

\subsection{Construction}

1. Setup $(d)$. The setup algorithm chooses a bilinear group $G$ of order $N=$ $p_{1} p_{2} p_{3}$ (where $p_{1}, p_{2}$, and $p_{3}$ are distinct primes). We let $G_{p_{i}}$ denote the subgroup of order $p_{i}$ in $G$. It chooses $g \in G_{p_{1}}$. Define the universe, $U$ of elements. For simplicity, we can take the first $|U|$ elements of $Z_{N}^{*}$ to be the universe. Namely, the integers $1, \ldots,|U| \bmod N$. Choose $t_{1}, \ldots, t_{|u|}$ uniformly at random from $Z_{N}$. Choose $y$ uniformly at random in $Z_{N}$. The published public parameters are:

$$
T_{1}=g^{t_{1}}, \ldots, T_{|U|}=g^{t_{|u|}}, \quad Y=e(g, g)^{y} .
$$

The master key is $t_{1}, \ldots, t_{|u|}, y$ and a generator of $G_{p_{3}}$.

2. KeyGeneration. To generate a private key for identity $w \subseteq U$ the following steps are taken. A $d-1$ degree polynomial $q$ is randomly chosen such that $q(0)=y$ and randomly $R_{i} \in G_{p_{3}}(i=1, \ldots, N)$ are chosen. The private key consists of components, $\left(D_{i}\right)_{i \in w}$, where $D_{i}=g^{\frac{q(i)}{t_{i}}} R_{i}$ for every $i \in w$.

3. Encryption. Encryption with the public key $w^{\prime}$ and message $M \in G_{2}$ proceeds as follows. First, a random value is chosen $s \in Z_{p}$ is chosen. The ciphertext is then published as:

$$
E=\left(w^{\prime}, E^{\prime}=M Y^{s},\left\{E_{i}=T_{i}^{s}\right\}_{i \in w^{\prime}}\right)
$$

Note that the identity, $w^{\prime}$, is included in the ciphertext.

4. Decryption. Suppose that a ciphertext, $E$, is encrypted with a key for identity $w^{\prime}$ and we have a private key for identity $w$, where $\left|w \cap w^{\prime}\right|>d$. Choose an arbitrary $d$-element subset, $S$, of $w \cap w^{\prime}$.

Then the ciphertext can be decrypted as

$$
\begin{aligned}
& \frac{E^{\prime}}{\prod_{i \in S}\left(e\left(D_{i}, E_{i}\right)\right)^{\delta_{i, s}(0)}} \\
& =\frac{E^{\prime}}{\prod_{i \in S}\left(e\left(g^{\frac{q_{i}}{t_{i}}} R_{i}, g^{t_{i} s}\right)\right)^{\delta_{i, s}(0)}} \\
& =\frac{E^{\prime}}{\prod_{i \in S} e(g, g)^{\delta_{i, s}(0) q_{i} s}} \\
& =\frac{E^{\prime}}{\prod_{i \in S} e(g, g)^{y s}} \\
& =M .
\end{aligned}
$$

The last equality is derived from using polynomial interpolation in the exponents. Since, the polynomial $s q(x)$ is of degree $d-1$ it can be interpolated using $d$ points. 


\subsection{Assumptions}

In this section, we give our complex assumption. These assumptions have been used in Lewko and Waters (2010b), Lewko et al. (2010).

Assumption 1 (Subgroup Decision Problem). Given $\left(N=p_{1} p_{2} p_{3}, G, G_{1}, e\right)$ select randomly $g \in G_{p_{1}}, X_{3} \in G_{p_{3}}, T_{1} \in G_{p_{1} p_{2}}, T_{2} \in G_{p_{1}}$ and set $D=\left(N, G, G_{1}, e, g, X_{3}\right)$. It is hard to distinguish $T_{1}$ from $T_{2}$. The advantage of an algorithm is defined as

$$
\operatorname{Adv}_{1}=\mid \operatorname{Pr}\left[A\left(D, T_{1}\right)=1\right]-\operatorname{Pr}\left[A\left(D, T_{2}\right)=1\right]
$$

Definition 3. Assumption 1 holds if $\mathrm{Adv}_{1}$ is negligible.

Assumption 2 Given $\left(N=p_{1} p_{2} p_{3}, G, G_{1}, e\right)$ choose randomly $g, X_{1} \in G_{p_{1}}, X_{2}, Y_{2} \in$ $G_{p_{2}}, X_{3}, Y_{3} \in G_{p_{3}}$, and set $D=\left(N, G, G_{1}, e, g, X_{1} X_{2}, X_{3}, Y_{2} Y_{3}\right)$. Then select $T_{1} \in G$, $T_{2} \in G_{p_{1} p_{3}}$ at random. It is hard to distinguish $T_{1}$ from $T_{2}$. The advantage of an algorithm is defined as

$$
\mathrm{Adv}_{2}=\mid \operatorname{Pr}\left[A\left(D, T_{1}\right)=1\right]-\operatorname{Pr}\left[A\left(D, T_{2}\right)=1\right]
$$

Definition 4. Assumption 2 holds if $\mathrm{Adv}_{2}$ is negligible.

Assumption 3 Given $\left(N=p_{1} p_{2} p_{3}, G, G_{1}, e\right)$, pick randomly $g \in G_{p_{1}}, X_{2}, Y_{2}, Z_{2} \in$ $G_{p_{2}}, X_{3} \in G_{p_{3}}, \alpha, s \in Z_{N}$ and set $D=\left(N, G, G_{1}, e, g, g^{\alpha} X_{2}, X_{3}, g^{s} Y_{2}, Z_{2}\right)$. Then compute $T_{1}=e(g, g)^{\alpha s}$ and pick randomly $T_{2} \in G_{1}$. It is hard to distinguish $T_{1}$ from $T_{2}$. The advantage of an algorithm is defined as

$$
\operatorname{Adv}_{3}=\mid \operatorname{Pr}\left[A\left(D, T_{1}\right)=1\right]-\operatorname{Pr}\left[A\left(D, T_{2}\right)=1\right]
$$

Definition 5. Assumption 3 holds if $\mathrm{Adv}_{3}$ is negligible.

\subsection{Security Proof}

In this section, we will prove the security of the proposed scheme. We first define semifunctional keys and semi-functional ciphertexts. Let $g_{2}$ denote a generator of $G_{p_{2}}$.

Semi-functional keys. At first, a normal secret key $\widehat{\left(D_{i}\right)}(i \in w)$ is generated by using the KeyGeneration algorithm. Then some random elements $\gamma_{i}(i \in w)$ are chosen in $Z_{N}$. The semi-functional keys are set as follows:

$$
D_{i}=\widehat{D_{i}} g_{2}^{\gamma_{i}}
$$

Semi-functional ciphertexts. At first, a normal ciphertext $\left(\widehat{w^{\prime}}, \widehat{E^{\prime}}, \widehat{E_{i}}\right)$ is obtained using the Encrypt algorithm. Then random elements $\lambda_{i}$ are chosen in $Z_{N}$. The semifunctional ciphertexts are set as follows:

$$
w^{\prime}=\widehat{w^{\prime}}, \quad E^{\prime}=\widehat{E^{\prime}}, \quad E_{i}=\widehat{E_{i}} g_{2}^{\lambda_{i}}
$$


We organize our proof as a sequence of games. The first game defined will be the real fuzzy identity-based encryption game and the last one will be one in which the adversary has no advantage unconditionally. We will show that each game is indistinguishable from the next (under three complexity assumptions). We first define the games as:

$\mathrm{Game}_{\text {real }}$ : This is a real FIBE security game. The next game, Game ${ }_{\text {restricated, will }}$ be like the real security game except that the attacker cannot ask for keys for identities which are equal to the challenge identity modulo $p_{2}$. This is a stronger restriction than the real security game, where the identities must be unequal modulo $N$. We will retain this stronger restriction throughout the subsequent games. The reason for it will be explained in the proof. For $0<i<q$, the $\mathrm{Game}_{i}$ is defined as follows.

Game $_{i}$ : Let $Q$ denote the set of private keys which the adversary queries during the games. This game is a real FIBE security game with the two exceptions: (1) The challenge ciphertext will be a semi-functional ciphertext. (2) The first $i$ keys will be semi-functional private keys. The rest of the keys in $Q$ will be normal.

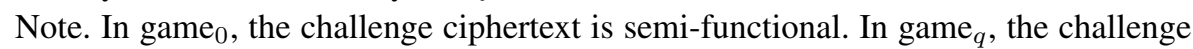
ciphertexts and all keys are semi-functional.

Game $_{\text {final }}$ : This game is the same with game ${ }_{q}$ except that the challenge ciphertext is a semi-functional encryption of random group element of $G_{1}$.

We will show that these games are indistinguishable in a set of lemmas. Let $\operatorname{Adv}_{\text {game }} \mathcal{A}$ denote the advantage in the real game.

Lemma 1. Suppose there exists an algorithm $\mathcal{A}$ such that Game $_{\text {Real }} \operatorname{Adv}_{\mathcal{A}}-$ Game $_{\text {Restricated }} \operatorname{Adv}_{\mathcal{A}}=\epsilon$.

Then we can build an algorithm $\mathcal{B}$ with advantage $>\frac{\epsilon}{2}$ in breaking either Assumption 1 or Assumption 2.

Proof. Given $g, X_{3}, \mathcal{B}$ can simulate Game Real with $\mathcal{A}$. With probability $\epsilon, \mathcal{A}$ produces identities ID and ID* such that ID $\neq I^{*}$ modulo $N$ and $p_{2}$ divides ID $-I^{*}$. $\mathcal{B}$ uses these identities to produce a nontrivial factor of $N$ by computing $a=\operatorname{gcd}\left(\right.$ ID - ID $\left.^{*}, N\right)$. We set $b=\frac{N}{a}$. We note that $p_{2}$ divides $a$ and $N=a b=p_{1} p_{2} p_{3}$. We consider two cases:

1. $p_{1}$ divides $b$.

2. $a=p_{1} p_{2}$ and $b=p_{3}$.

At least one of these cases must occur with probability $>\frac{\epsilon}{2}$. In case $1, \mathcal{B}$ will break assumption 1 . Given $g, X_{3}, T, \mathcal{B}$ can determine that $p_{1}$ divides $b$ by verifying that $g^{b}$ is the identity and will then test whether $T^{b}$ is the identity. If it is, then $T \in G_{p_{1}}$. If it is not, $T \in G_{p_{1} p_{2}}$.

In case 2, $\mathcal{B}$ will break Assumption 2. Given $g, X_{1} X_{2}, X_{3}, Y_{2} Y_{3}$, B can determine that $a=p_{1} p_{2}$ by verifying that $\left(X_{1} X_{2}\right)^{a}$ is the identity and will then test whether $e\left(\left(Y_{2} Y_{3}\right)^{b}, T\right)$ is the identity. If it is, then $T \in G_{p_{1} p_{3}}$. If it is not, then $T \in G$.

Lemma 2. Suppose that there exists an algorithm $\mathcal{A}$ such that

$\operatorname{Adv}_{\text {game }_{\text {Restricated }}} \mathcal{A}-\operatorname{Adv}_{\text {game }_{0}} \mathcal{A}=\epsilon$.

Then we can build an algorithm $\mathcal{B}$ with advantage $\epsilon$ in breaking Assumption 1. 
Proof. Our algorithm $\mathcal{B}$ begins by receiving $g, X_{3}, T$ where $g \in G_{p_{1}}, X_{3} \in G_{p_{3}}$. It works as follows:

1. Setup. $\mathcal{B}$ chooses random elements $t_{1}, \ldots, t_{|U|}, y \in Z_{N}$ and sets $T_{i}=g^{t_{i}}$, $Y=e(g, g)^{y}$ for $1<i<|U|$. It sends the public keys $P K=\left(T_{1}, \ldots, T_{|U|}\right.$, $\left.Y=e(g, g)^{y}\right)$ to $\mathcal{A}$.

2. Query phase 1 . The adversary $\mathcal{A}$ issues a private key query for identity $w$. B answers as follows: A $d-1$ degree polynomial $q$ is randomly chosen such that $q(0)=y$ and $t_{i}^{\prime}(i \in w)$ are randomly chosen in $Z_{N}$. Then it sets

$$
D_{i}=g^{\frac{q(i)}{t_{i}}} X_{3}^{t_{i}^{\prime}}
$$

It is a valid simulation to $\mathcal{A}$.

3. Challenge. The adversary $\mathcal{A}$ outputs two challenge message $M_{0}, M_{1}$ and a challenge identity $w^{*}$. Then the ciphertext is formed as

$$
w^{*}, \quad E^{\prime}=M_{b} e(T, g)^{y}, \quad E_{i}=T^{t_{i}}\left(i \in w^{*}\right)
$$

where $b \in\{0,1\}$.

4. Query phase 2. The adversary continues to issue queries $q_{j}$, where $q_{j}$ is the following:

- Extraction query $(\gamma)$ : as in phase 1 with the constraint that $\left|\gamma \cap w^{*}\right|<d$.

5. Guess. Finally, the adversary $\mathcal{A}$ outputs a guess $b^{\prime} \in\{0,1\}$ and wins the game if $b^{\prime}=b$.

If $T \in G_{p_{1} p_{2}}$, then $\left(w^{*}, E^{\prime}, E_{i}\left(1<i<\left|w^{*}\right|\right)\right)$ is a semi-functional ciphertext. If $T \in G_{p_{1}}$, then $\left(w^{*}, E^{\prime}, E_{i}\left(1<i<\left|w^{*}\right|\right)\right)$ is a normal ciphertext. Hence $\mathcal{B}$ can use $\mathcal{A}$ 's guess to break Assumption 1 with advantage $\epsilon$.

Lemma 3. Suppose there exists an algorithm $\mathcal{A}$ such that Game $_{k-1} \mathrm{Adv}_{\mathcal{A}}-$ Game $_{k} \mathrm{Adv}_{\mathcal{A}}=\epsilon$.

Then we can build an algorithm $\mathcal{B}$ with advantage $\epsilon$ in breaking Assumption 2.

Proof. $\mathcal{B}$ first receives $g, X_{1} X_{2}, X_{3}, Y_{2} Y_{3}, T$. It works as follows:

1. Setup. $\mathcal{B}$ chooses random elements $t_{1}, \ldots, t_{|U|}, y \in Z_{N}$ and sets $T_{i}=g^{t_{i}}, Y=$ $e(g, g)^{y}$ for $1<i<|U|$. It sends the public keys $P K=\left(T_{1}, \ldots, T_{|U|}, Y=\right.$ $\left.e(g, g)^{y}\right)$ to $\mathcal{A}$.

2. Query phase 1.

(a) The adversary $\mathcal{A}$ issues the $i t h$ private key query for identity $w$ when $i<k$. $\mathcal{B}$ answers as follows: two $d-1$ degree polynomial $q_{1}, q_{2}$ is randomly chosen such that $q_{1}(0)=y, q_{2}(0)=0$. Then it sets

$$
D_{i}=g^{\frac{q_{1}(i)}{t_{i}}}\left(Y_{2} Y_{3}\right)^{\frac{q_{2}(i)}{t_{i}}}
$$


It is a semi-functional key to $\mathcal{A}$.

(b) The adversary $\mathcal{A}$ issues the $i t h$ private key query for identity $w$ when $i>k$. $\mathcal{B}$ answers as follows: two $d-1$ degree polynomial $q_{1}, q_{2}$ is randomly chosen such that $q_{1}(0)=y, q_{2}(0)=0$. Then it sets

$$
D_{i}=g^{\frac{q_{1}(i)}{t_{i}}}\left(X_{3}\right)^{\frac{q_{2}(i)}{t_{i}}} .
$$

It is a normal key to $\mathcal{A}$.

(c) The adversary $\mathcal{A}$ issues the $i$ th private key query for identity $w$ when $i=k$. $\mathcal{B}$ answers as follows: two $d-1$ degree polynomial $q_{1}, q_{2}$ is randomly chosen such that $q_{1}(0)=y, q_{2}(0)=0$. Then it sets

$$
D_{i}=g^{\frac{q_{1}(i)}{t_{i}}}(T)^{\frac{q_{2}(i)}{t_{i}}} .
$$

3. Challenge. The adversary $\mathcal{A}$ outputs two challenge message $M_{0}, M_{1}$ and a challenge identity $w^{*}$. Then the ciphertext is formed as

$$
w^{*}, \quad E^{\prime}=M_{b} e\left(X_{1} X_{2}, g\right)^{y}, \quad E_{i}=\left(X_{1} X_{2}\right)^{t_{i}}\left(i \in w^{*}\right),
$$

where $b \in\{0,1\}$.

If $\mathcal{B}$ attempts to test itself whether key $k$ is semi-functional by creating a semifunctional ciphertext for $w^{\prime}\left(\left|S=\left\{w^{\prime} \cap w\right\}\right|>d\right)$ and trying to decrypt, then decryption will work whether key $k$ is semi-functional or not, because

$$
\begin{aligned}
& \prod_{i \in S}\left(e\left(D_{i}, E_{i}\right)\right)^{\delta_{i, s}(0)} \\
& \quad=\frac{E^{\prime}}{\prod_{i \in S}\left(e\left(g^{\frac{q_{1}(i)}{t_{i}}}(T)^{\frac{q_{2}(i)}{t_{i}}},\left(X_{1} X_{2}\right)^{t_{i}}\right)\right)^{\delta_{i, s}(0)}} \\
& \quad=\frac{E^{\prime}}{\prod_{i \in S} e(g, g)^{\delta_{i, s}(0) q_{1}(i) s} e\left(g_{2}, g_{2}\right)^{\delta_{i, s}(0) q_{2}(i) s}} \\
& \quad=\frac{E^{\prime}}{\prod_{i \in S} e(g, g)^{y s} e\left(g_{2}, g_{2}\right)^{0}} \\
& \quad=M .
\end{aligned}
$$

In other words, the simulator $\mathcal{B}$ can only make a nominally semi-functional key $k$.

4. Query phase 2 . The adversary continues to issue queries $q_{j}$, where $q_{j}$ is the following:

- Extraction query $(\gamma)$ : as in phase 1 with the constraint that $\left|\gamma \cap w^{*}\right|<d$.

5. Guess. Finally, the adversary $\mathcal{A}$ outputs a guess $b^{\prime} \in\{0,1\}$ and wins the game if $b^{\prime}=b$.

If $T \in G_{p_{1} p_{3}}$, then $\mathcal{B}$ has properly simulated $\mathrm{Game}_{k-1}$. If $T \in G$, then $\mathcal{B}$ has properly simulated $\mathrm{Game}_{k}$. Hence $\mathcal{B}$ can use the output of $\mathcal{A}$ to break Assumption 2 with advantage $\epsilon$. 
Lemma 4. Suppose there exists an algorithm $\mathcal{A}$ such that

Game $_{k-1} \operatorname{Adv}_{\mathcal{A}}-$ Game $_{k} \operatorname{Adv}_{\mathcal{A}}=\epsilon$.

Then we can build an algorithm $\mathcal{B}$ with advantage $\epsilon$ in breaking Assumption 3.

Proof. $\mathcal{B}$ first receives $g, g^{\alpha} X_{2}, X_{3}, g^{s} Y_{2}, Z_{2}, T$. It works as follows:

1. Setup. $\mathcal{B}$ chooses random elements $t_{1}, \ldots, t_{|U|}, y \in Z_{N}$ and sets $T_{i}=g^{t_{i}}, Y=$ $e\left(g^{\alpha} X_{2}, g\right)$ for $1<i<|U|$. It sends the public keys $P K=\left(T_{1}, \ldots, T_{|U|}, Y\right)$ to $\mathcal{A}$.

2. Query phase 1. The adversary $\mathcal{A}$ issues a private key query for identity $w$. B answers as follows: A $d-1$ degree polynomial $q$ is randomly chosen such that $q(0)=y$ and $t_{i}^{\prime}(i \in w)$ are randomly chosen in $Z_{N}$. Then it sets

$$
D_{i}=g^{\frac{q(i)}{t_{i}}} X_{3}^{t_{i}^{\prime}}
$$

It is a valid simulation to $\mathcal{A}$.

3. Challenge. The adversary $\mathcal{A}$ outputs two challenge message $M_{0}, M_{1}$ and a challenge identity $w^{*}$. Then the ciphertext is formed as

$$
w^{*}, \quad E^{\prime}=M_{b} T, \quad E_{i}=\left(g^{s} Y_{2}\right)^{t_{i}}\left(i \in w^{*}\right),
$$

where $b \in\{0,1\}$.

4. Query phase 2. The adversary continues to issue queries $q_{j}$, where $q_{j}$ is the following:

- Extraction query $(\gamma)$ : as in phase 1 with the constraint that $\left|\gamma \cap w^{*}\right|<d$.

5. Guess. Finally, the adversary $\mathcal{A}$ outputs a guess $b^{\prime} \in\{0,1\}$ and wins the game if $b^{\prime}=b$.

If $T=e(g, g)^{\alpha s}$, then $\left(w^{*}, E^{\prime}, E_{i}\left(1<i<\left|w^{*}\right|\right)\right)$ is a semi-functional ciphertext. then this is a properly distributed semi-functional ciphertext with message $M_{b}$. If $T$ is a random element of $G_{T}$, then this is a semi-functional ciphertext with a random message. Hence $\mathcal{B}$ can use $\mathcal{A}$ 's guess to break Assumption 3 with advantage $\epsilon$.

Theorem 1. If Assumptions 1, 2, and 3 hold, then our FIBE system is fully IND-FID-CPA secure.

Proof. If Assumptions 1, 2, and 3 hold, then we have shown by the previous lemmas

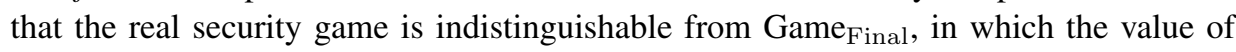
$b$ is information theoretically hidden from the attacker. Hence the attacker can attain no advantage in breaking the FIBE system.

\section{Conclusion}

In this paper, we analyzed Ren et al.'s (2010) FIBE scheme. We first show their scheme is not correct and then we give a resist collusion attack for the scheme's key generation 
process. At last, we propose a new fully secure FIBE scheme by using the "dual system encryption" technique (Waters, 2009) and prove its security.

Acknowledgments. The authors would like to express their gratitude thanks to the anonymous reviewer for pointing out the incorrectness of Ren et al.'s FIBE scheme, they also thanks Dr. Jian Weng for valuable comments. This work is supported by the National Natural Science Foundation of China under contract no. 61103230, 61003232, 61103231, Natural Science Foundation of Shaanxi Province under contract no. 2010JM8034, 2011JM8012.

\section{References}

Baek, J., Susilo, W., Zhou, J. (2007). New constructions of fuzzy identity-based encryption. In: Proceedings of ACM Symposium on Information, Computer and Communication Security, pp. 368-370.

Boneh, D., Boyen, X. (2004a). Efficient selective-id secure identity based encryption without random oracles. In: Proceedings of EUROCRYPT 2004, Lecture Notes in Computer Science, pp. 223-238.

Boneh, D., Boyen, X. (2004b). Secure identity based encryption without random oracles. In: Proceedings of CRYPTO 2004, Lecture Notes in Computer Science, pp. 443-459.

Boneh, D., Franklin, M. (2001). Identity based encryption from the Weil pairing. In: Proceedings of CRYPTO 2001, Lecture Notes in Computer Science, pp. 213-229.

Canetti, R., Halevi, S., Katz, J. (2003). A forward-secure public-key encryption scheme. In: Proceedings of EUROCRYPT 2003, Lecture Notes in Computer Science, pp. 255-271.

Gentry, C. (2006). Practical identity-based encryption without random oracles. In: Proceedings of EUROCRYPT 2006, Lecture Notes in Computer Science, pp. 445-464.

Goyal, V., Pandey, O., Sahai, A., Waters, B. (2006). Attribute-based encryption for fine-grained access control of encrypted data. In: Proceedings of ACM Symposium on Information, Computer and Communication Security, pp. 89-98.

Lewko, A., Waters, B. (2010). New techniques for dual system encryption and fully secure hibe with short ciphertexts. In: Proceedings of TCC 2010, Lecture Notes in Computer Science, pp. 455-479.

Lewko, A., Okamoto, T., Sahai, A., Takashima, K., Waters, B. (2010). Fully secure functional encryption: Attribute-based encryption and (hierarchical) inner product encryption. In: Proceedings of EUROCRYPT 2010, Lecture Notes in Computer Science, pp. 62-91.

Pirretti, M., Traynor, P., McDaniel, P., Waters, B. (2006). Secure attribute-based systems. In: Proceedings of ACM Symposium on Information, Computer and Communication Security, pp. 99-112.

Ren, Y., Gu, D., Wang, S., Zhang, X. (2010). New fuzzy identity-based encryption in the standard model. Informatica, 21(3), 393-407.

Sahai, A., Waters, B. (2005). Fuzzy identity-based encryption. In: Proceedings of EUROCRYPT2005, Lecture Notes in Computer Science, pp. 457-473.

Shamir, A. (1984). Identity-based cryptosystems and signature Schemes. In: Proceedings of CRYPTO 1984, Lecture Notes in Computer Science, pp. 47-53.

Waters, B. (2005). Efficient identity-based encryption without random oracles. In: Proceedings of EUROCRYPT 2005, Lecture Notes in Computer Science, pp. 114-127.

Waters, B. (2009). Dual system encryption: realizing fully secure ibe and hibe under simple assumptions. In: Proceedings of CRYPTO 2009, Lecture Notes in Computer Science, pp. 619-636. 
X. Wang was born in 1981. He obtained his MS and BS degrees from the Engineering University of Chinese Armed Police Force. Now he is a senior lecturer in the same college, his main research fields are cryptography and information security.

X. Yang was born in 1959. He obtained his MS and BS degrees from the Xidian University. Now he is a professor in the Engineering University of Chinese Armed Police Force. His main research fields include cryptography, information hiding and security.

M. Zhang was born in 1969. She obtained her MS degree from the the Northwestern Polytechnical University, and her BS degree from Engineering University of Chinese Armed Police Force. Now she is a professor in the Engineering University of Chinese Armed Police Force. Her main research fields are information security and database management.

Y. Yu was born in 1981. He obtained his Phd degree from the Xidian University in 2008. Now he is an associate professor in the University of Electronic Science and Technology of China. His main research fields include cryptography and information security.

\section{Standartinio modelio tapatumu gristas neraiškiosios šifravimo schemos analizè}

Xu An WANG, Xiaoyuan YANG, Minqing ZHANG, Yong YU

Tapatumu grịstas neraiškusis šifravimas (FIBE), kurị pasiūle Sahai ir Waters, yra naujas tapatumu grịstas šifravimo metodas. Jis igalina vartotoją, kurio tapatybè $w$, dešifruoti tekstą užšifruota viešuoju raktu $w^{\prime}$, jei ir tik jei vartotojų identifikatoriai ID ir ID' mažai skiriasi. Neseniai Ren ir kt. pasiūlè naują FIBE schemą ir tvirtino, kad ji yra visiškai saugi. Šiame straipsnyje parodyta, kad ju schema nèra gera, o ju schemos rakto generavimo procesas nèra atsparus konfliktinèms atakoms. Pasiūlyta nauja visiškai saugi FIBE schema besiremianti Sahai-Waters FIBE schema, o jos saugumas ịrodytas naudojant ,dvigubo šifravimo“ metodą. 